\title{
The future is near: Universally usable mainstream on-line publishing
}

\author{
John A. Gardner ${ }^{\mathrm{a}, \mathrm{b}}$ and Robert A. Kelly ${ }^{\mathrm{c}}$ \\ ${ }^{a}$ President, View Plus Technologies, Corvallis, OR, USA \\ ${ }^{\mathrm{b}}$ Professor Emeritus, Oregon State University, Corvallis, OR, USA \\ ${ }^{\mathrm{c}}$ Director, Journal Information Systems, The American Physical Society, Ridge, NY, USA
}

John Gardner is blind and he is the founder of ViewPlus Technologies, a company whose mission is dedicated to information accessibility. He is also professor emeritus of physics. It was a very natural thing for him to collaborate with the American Physical Society. Physicist lead! "We will show something that's very exciting in this presentation". It's particularly exciting for people who are blind and have print disabilities in particular. $25 \%$ or more of the world population have print disabilities - i.e. they have difficulty grasping regular print information. They are helped if they can see the print and also hear it and feel it. These people are largely being eliminated from sciences. This disability is not correlated with intelligence or ability to learn. It is simply that they have visual processing difficulties. So by making something accessible to blind people and people with print disabilities, we are actually increasing the number of potential scientist by a significant fraction.

A video shows what we could demonstrate in real time in a longer session. The video transcript follows, and the video itself is included with the proceedings archives:

"ViewPlus Technologies is working together with the American Physical Society, APS, to publish journals that include fully accessible text, math equations and graphics allowing all readers to have access to the same information. Creating these materials is a two step process: The first step is to convert the text and math equations into an accessible format that can be read using most DAISY-players and screen readers. ViewPlus works together with Design Science on this part of the project. The second step involved is to convert graphics into a scalable vector graphic, SVG format for use with the IVEO Hands-on learning system, developed by ViewPlus. This second step gives users access to all of the graphical information to touch, sound and sight with the availability to zoom in or out for more or less detail. Here is how it works: You can open an APS journal with any modern DAISY-player or within a standard web browser and navigate using a screen reader. With the file open you can explore the document as you normally would. As you come across complex math-equations they are read with perfect pronunciations. [...] Each graphic within the document includes a link to the SVG file and a caption describing the image. As you come to a graphic, the link is read first followed by the caption. [...] To explore in greater detail, select the specified link. The graphic will then open in IVEO Hands-on learning system. To utilize the full power of IVEO, first print the document to your Tiger Embosser for a high resolution tactile printout. Then place the printout on the IVEO touch-sensitive tablet. You can then touch different areas of the graphic and have a description spoken back to you. [...] This gives users access to an unlimited demand of information for more complete explanation of the image. Once you have finished exploring the graphic you can then easily navigate back to the journal and continue reading 
with ease. As you can see ViewPlus and the American Physical Society have for the first time found a way to include text, complex math-equations, and graphics within published materials for unlimited access for all. To learn more about the IVEO Hands-on learning system and ViewPlus Tiger Embossers visit: www.viewplus.com".

Robert Kelly, with the American Physical Society, notes that for most people, the screen reader voice is hard to understand. Screen readers are configurable to match users' preferences - voice pitch, gender, speed. He recalls a demonstration back in the 1980s by a blind programmer, reading an IBM COBOL manual and the IBM BookManager product, "he had this thing reading at a speed that I could not understand. It was almost like: "Zzz. .." but he was able to navigate through the IBM manual in a way that I could not. This was due to using the tags in the manual for navigation. So just because an untrained person cannot instantly use this technology does not mean that it cannot be learned". One more movie is now shown. Its transcript:

"IVEO Hands-on learning system teaches using touch, sound, and sight combined. This method utilizes any or all three learning modalities to help students learn faster and retain information longer, meanwhile making learning more fun and interactive. IVEO is a great tool to use to teach difficult subjects, such as biology, health, chemistry, astronomy, math and geography - the possibilities are endless. You can use premade curriculum or can create your own using any mainstream graphics software, such as Adobe Illustrator or Corel Draw. You can also use powerful authoring tools in the IVEO creator software giving you the flexibility to customize your lesson plans. Once you decided which method to use, open the file in IVEO, and print the document using your ViewPlus Embosser. The example shown is being printed using the Emprint SpotDot Color Ink and Braille Embosser. You can also choose to use an ink-printer if the tactile output is not necessary. Once the document has finished printing, take the print out and place it on the IVEO touchpad, using the clamp to secure the document in place. Begin pressing different areas of the graphic to hear the titles spoken back to you. Within IVEO you have the option of including multiple levels of information. You can hear a longer description of the object, if more information is necessary. Not to mention you can attach sound files or record your own voice to further stimulate learning and help students to make the connection between the object they are touching and the presence in everyday life. IVEO also allows you to scale your document to any size. You can zoom in or out for more or less detail. You can then reprint the document and place it on the touchpad, giving you access to all the information, no matter the size. This universal teaching system makes learning more complete for all students, while also including tools necessary for people with visual and learning disabilities. For more information about the IVEO Hands-on learning system visit www.viewplus.com".

As you can probably tell from the video, the IVEO Hands-on learning system is primarily being marketed now for use in lower grade education and initially for blind students and dyslexic students. But in fact ViewPlus testers find that all little kids love it. So it's really an educational tool. The part that will be used for displaying figures from physical review, physical review letters, etc. is a generalisation of IVEO. It was designed from the beginning to be generalised. It's intended to be a technology that allows essentially any kind of static graphical information to be created, displayed, and distributed in a form that is fully accessible to people who are blind or dyslexic. People who are blind or dyslexic may need some additional hardware such as a touchpad and may need a tactile copy. Tactile copies are made with hardware that is - guess what - built by ViewPlus. This is why the company was founded - to make it possible to do this. Those tactile copies can also be ink-printed with gray scale images using the ViewPlus Pro Ink Embosser or in color with the ViewPlus Emprint SpotDot. These are useful for many people who have visual processing difficulties such as dyslexia. 